\title{
Successive Approximation Method for Solving Nonlinear Diffusion Equation with Convection Term
}

\author{
${ }^{1}$ Saad A. Manaa, ${ }^{2}$ Fadhil H. Easif, ${ }^{3}$ Bewar A. Mahmood \\ ${ }^{1,2,3}$ Department of Mathematics, Faculty of Science, University of Zakho,Duhok, Kurdistan Region, Iraq
}

\begin{abstract}
Nonlinear diffusion equation with convection term solved numerically using successive approximation method. Numerical example showed that (SAM) can solve this kind of models also comparing with the exact solution showed that SAM accurate and efficient method as shown in table (1) and Figures $(1,2)$.
\end{abstract}

Keywords: - Diffusion equation with convection term, Successive approximation method.

\section{INTRODUCTION}

This method starts by using the constant function as an approximation to a solution. We substitute this approximation into the right side of the given equation and use the result as a next approximation to the solution. Then we substitute this approximation into the right side of the given equation to obtain what we hope is a still better approximation and we continuing the process. Our goal is to find a function with the property that when it is substituted in the right side of the given equation the result is the same function. This procedure is known as successive approximation method Nowadays engineers and scientists in all fields of their research are using partial differential equations to describe their problems and thus such partial differential equations arise in the study of heat transfer, boundary-layer flow, fluid flow problems, vibrations elasticity, circular and rectangular wave guides, in applied mathematics and so on.[1]

Many physical, chemical and engineering problems mathematically can be modeled in the form of system of partial difference equations or system of ordinary difference equations. Finding the exact solution for the above problems which involve partial differential equations is difficult in some cases. Hence we have to find the numerical solution of these problems using computers which came into existence. [2]

\section{I.1 MATHEMATICAL MODEL}

We consider the nonlinear diffusion equation with convection term of the form:

$u_{t}=\left(A(u) u_{x}\right)_{x}+B(u) u_{x}+C(u)$

Where $\mathrm{u}=\mathrm{u}(\mathrm{x}, \mathrm{t})$ is the unknown function and $A(u), B(u)$, and $C(u)$ are arbitrary smooth functions on $\mathrm{u}$. The indices $t$ and $x$ denotes differentiating with respect to the variables. The study of solution of this problem has over the years attracted the interest of many researches. Chemiha and Serov [3] considered the lie and non-lie symmetries of non linear diffusion with convection term. In this work, we apply successive approximations method (SAM) to approximate solution of the generalized non linear diffusion equation with convection term of the form:

$u_{t}=a u_{x x}+b u u_{x}+c u(u-k)(u+k)$

with taking $A(u)=a, B(u)=b u$, and $C(u)=c u(u-k)(u+k)$

with the initial condition $u(x, 0)=f(x), \quad \alpha \leq x \leq \beta$

\section{MATERIALS AND METHODS}

II.1 Basic concepts of successive approximations method (SAM) [4] and [5]

The method of successive approximations is one of the powerful methods for solving partial differential equations. The method of SAM provides a method that can, in principle, be used to solve any initial value problem:

$u^{\prime}=f(u, t)$ be any initial value problem with initial condition $u\left(t_{0}\right)=u_{0}$

Now, integrating both sides of equation (2) with respect to $t$ on the interval $(0, t)$, we obtain:

$u(t)=u_{0}+\int_{t_{0}}^{t} f(u(s), s) d s$

and then iteratively constructs a sequence of solutions that get closer and closer to the actual (exact) solutions of

(2). The SAM is based on the integral equation (3) as follows:

$u_{0}(t)=u_{0}$

$u_{1}(t)=u_{0}+\int_{t_{0}}^{t} f\left(u_{0}, s\right) d s$ 
$u_{2}(t)=u_{0}+\int_{t_{0}}^{t} f\left(u_{1}, s\right) d s$

$u_{3}(t)=u_{0}+\int_{t_{0}}^{t} f\left(u_{2}, s\right) d s$

This process can be continued to obtain the $n^{\text {th }}$ approximation,

$u_{n}(t)=u_{0}+\int_{t_{0}}^{t} f\left(u_{n-1}(s), s\right) d s \quad, \quad n=1,2, \ldots$

Then determine whether $u(x)$ approaches the solution $u(x)$ as $\mathrm{n}$ increases. This will be done by proving the following:

- The sequence $\{u(x)\}$ converges to a limit $u(x)$, that

$\lim _{n \rightarrow \infty} u_{n}(x)=u(x) \quad a \leq x \leq b$.

- The limiting function $u(x)$ is a solution of (3) on the interval $a \leq x \leq b$.

- The solution $u(x)$ of (3) is unique.

A proof of these results can be constructed along the lines of the corresponding proof for ordinary differential equations (see[6]). And this process is called Successive Approximations Method.

\section{II.2 Derivation of SAM for Solving Nonlinear Diffusion Equation with Convection Term}

The general successive approximation method for equation (1) is in the form:

$$
\begin{aligned}
u_{n}(x, t)=u(x, 0) & +\int_{0}^{t} a \frac{\partial^{2} u_{n-1}(x, s)}{\partial x^{2}} d s+\int_{0}^{t} b u_{n-1}(x, s) \frac{\partial u_{n-1}(x, s)}{\partial x} d s \\
& +\int_{0}^{t} c u_{n-1}(x, s)\left(\left(u_{n-1}(x, s)-k\right)\left(u_{n-1}(x, s)+k\right)\right) d s
\end{aligned}
$$

To approximate solution for equation (1), we start with putting $n=1$ in equation (9) to obtain $u_{1}(x, t)$

$$
\begin{aligned}
& u_{1}(x, t)=u_{0}(x, t)+\int_{0}^{t} a \frac{\partial^{2} u_{0}(x, s)}{\partial x^{2}} d s+\int_{0}^{t} b u_{0}(x, s) \frac{\partial u_{0}(x, s)}{\partial x} d s \\
& +\int_{0}^{t} c u_{0}(x, s)\left(\left(u_{0}(x, s)-k\right)\left(u_{0}(x, s)+k\right)\right) d s \\
& u_{1}(x, t)=u_{0}(x, t)+\left(a \frac{\partial^{2} u_{0}(x, t)}{\partial x^{2}}+b u_{0}(x, t) \frac{\partial u_{0}(x, t)}{\partial x}+c\left(u_{0}^{3}(x, t)-k^{2} u_{0}(x, t)\right)\right) t \\
& \text { Let } w_{0}(x, t)=a \frac{\partial^{2} u_{0}(x, t)}{\partial x^{2}}+b u_{0}(x, t) \frac{\partial u_{0}(x, t)}{\partial x}+c\left(u_{0}{ }^{3}(x, t)-k^{2} u_{0}(x, t)\right) \\
& \text { So } u_{1}(x, t)=u_{0}(x, t)+w_{0}(x, t) t
\end{aligned}
$$

Put $n=2$ in equation (9) to obtain a second approximation $u_{2}(x, t)$ as follows:

$u_{2}(x, t)=u_{0}(x, t)+\int_{0}^{t} a \frac{\partial^{2} u_{1}(x, s)}{\partial x^{2}} d s+\int_{0}^{t} b u_{1}(x, s) \frac{\partial u_{1}(x, s)}{\partial x} d s+\int_{0}^{t} c\left(u_{1}{ }^{3}(x, s)-k^{2} u_{1}(x, s)\right) d s$

Substituting equation (11) in equation (12) , we get

$$
\begin{aligned}
u_{2}(x, t)=u_{0}(x, t) & +\int_{0}^{t} a \frac{\partial^{2}}{\partial x^{2}}\left(u_{0}(x, s)+w_{0}(x, s) s\right) d s+\int_{0}^{t}\left[b ( u _ { 0 } ( x , s ) + w _ { 0 } ( x , s ) s ) \frac { \partial } { \partial x } \left(u_{0}(x, s)\right.\right. \\
& \left.\left.+w_{0}(x, s) s\right)\right] d s+\int_{0}^{t} c\left(\left(u_{0}(x, s)+w_{0}(x, s) s\right)^{3}-k^{2}\left(u_{0}(x, s)+w_{0}(x, s) s\right)\right) d s \\
u_{2}(x, t)=u_{0}(x, t)+ & \left(a \frac{\partial^{2} u_{0}(x, t)}{\partial x^{2}}+b u_{0}(x, t) \frac{\partial u_{0}(x, t)}{\partial x}+c u_{0}^{3}(x, t)-c k^{2} u_{0}(x, t)\right) t \\
& +\left(a \frac{\partial^{2} w_{0}(x, t)}{\partial x^{2}} b u_{0}(x, t) \frac{\partial w_{0}(x, t)}{\partial x}+b w_{0}(x, t) \frac{\partial u_{0}(x, t)}{\partial x}+3 c u_{0}{ }^{2}(x, t) w_{0}(x, t)\right. \\
- & \left.c k^{2} w_{0}(x, t)\right) \frac{t^{2}}{2}+\left(b w_{0}(x, t) \frac{\partial w_{0}(x, t)}{\partial x}+3 c u_{0}(x, t) w_{0}{ }^{2}(x, t)\right) \frac{t^{3}}{3}+c w_{0}{ }^{3}(x, t) \frac{t^{4}}{4}
\end{aligned}
$$

By the same way for $\mathrm{n}=3,4 \ldots$

\section{NUMERICAL APPLICATIONS}

We will apply Successive Approximations Method (SAM) to solve the nonlinear diffusion equation with convection term, and present numerical results to verify the effectiveness of these methods, we take the following example: 


\section{III.1. Numerical Example and results}

In this section, we present example of nonlinear diffusion equation with convection term and results will be compared with the exact solutions.

Consider the following nonlinear diffusion equation with convection term.[7]

$u_{t}=a u_{x x}+b u u_{x}+\frac{b^{2}}{9 a} u(u-k)(u+k)$

With the initial condition

$u(x, o)=\frac{k\left(-1+c_{1} e^{\frac{b k x}{3 a}}\right)}{1+c_{1} e^{\frac{b k x}{3 a}}+c_{2} e^{\frac{b k x}{6 a}}}$

and boundary conditions

$$
u(0, t)=\frac{k\left(-1+c_{1}\right)}{1+c_{1}+c_{2} e^{\frac{b^{2} k^{2} t}{12 a}}} \quad \text {, and } u(1, t)=\frac{k\left(-1+c_{1} e^{\frac{b k}{3 a}}\right)}{1+c_{1} e^{\frac{b k}{3 a}}+c_{2} e^{\frac{b^{2} k^{2} t}{12 a}+\frac{b k}{6 a}}}
$$

Where $\mathrm{a} \neq 0, \mathrm{~b}$ and $\mathrm{k}$ are arbitrary constants. In this example, $(u)=a, B(u)=b u$, and $c(u)=\frac{b^{2}}{9 a} u(u-k)(u+k)$

The exact solutions of this equation have been derived by Andrei D. Polyanin and Valentin F. Zaitsev .[7]

$$
u(x, t)=\frac{k\left[-1+c_{1} e^{\frac{b k x}{3 a}}\right]}{1+c_{1} e^{\frac{b k x}{3 a}}+c_{2} e^{\frac{b^{2} k^{2} t}{12 a}+\frac{b k x}{6 a}}}
$$

Where $c_{1}$ and $c_{2}$ are arbitrary constants.

Apply the SAM and by using Matlab, we obtained $u_{1}$ and $u_{2}$ as follows:

$$
\begin{aligned}
& u_{0}(x, t)=\frac{k\left(-1+c_{1} e^{\frac{b k x}{3 a}}\right)}{1+c_{1} e^{\frac{b k x}{3 a}}+c_{2} e^{\frac{b k x}{6 a}}} \\
& u_{1}(x, t)=\frac{k\left(-1+c_{1} e^{\frac{b k x}{3 a}}\right)}{1+c_{1} e^{\frac{b k x}{3 a}}+c_{2} e^{\frac{b k x}{6 a}}}-\frac{b^{2} e^{\frac{b k x}{6 a}} k^{3} t\left(-1+c_{1} e^{\frac{b k x}{3 a}}\right) c_{2}}{12 a\left(1+c_{1} e^{\frac{b k x}{3 a}}+c_{2} e^{\frac{b k x}{6 a}}\right)^{2}} \\
& u_{2}(x, t)=\frac{k\left(-1+c_{1} e^{\frac{b k x}{3 a}}\right)}{\left(1+c_{1} e^{\frac{b k x}{3 a}}+c_{2} e^{\frac{b k x}{6 a}}\right)} \\
& -\left\{b ^ { 2 } e ^ { \frac { b k x } { 6 a } } k ^ { 3 } t ( - 1 + c _ { 1 } e ^ { \frac { b k x } { 3 a } } ) c _ { 2 } \left(5184 a^{3}+20736 a^{3} c_{1} e^{\frac{b k x}{3 a}}+20736 a^{3} c_{2} e^{\frac{b k x}{6 a}}\right.\right. \\
& +20736 a^{3} c_{1}{ }^{3} e^{\frac{b k x}{a}}+31104 a^{3} c_{1}{ }^{2} e^{\frac{2 b k x}{3 a}}+31104 a^{3} c_{2}{ }^{2} e^{\frac{b k x}{3 a}}+20736 a^{3} c_{2}{ }^{3} e^{\frac{b k x}{2 a}} \\
& +5184 a^{3} c_{2}{ }^{4} e^{\frac{2 b k x}{3 a}}+5184 a^{3} c_{1}{ }^{4} e^{\frac{4 b k x}{3 a}}+62208 a^{3} c_{1} c_{2}{ }^{2} e^{\frac{2 b k x}{3 a}}+62208 a^{3} c_{1}{ }^{2} c_{2} e^{\frac{5 b k x}{6 a}} \\
& +20736 a^{3} c_{1} c_{2}{ }^{3} e^{\frac{5 b k x}{6 a}}+20736 a^{3} c_{1}{ }^{3} c_{2} e^{\frac{7 b k x}{6 a}}+216 a^{2} k^{2} b^{2} t+31104 a^{3} c_{1}{ }^{2} c_{2}{ }^{2} e^{\frac{b k x}{a}} \\
& +62208 a^{3} c_{1} c_{2} e^{\frac{b k x}{2 a}}+b^{6} c_{2}{ }^{2} k^{6} t^{3} e^{\frac{b k x}{3 a}}+b^{6} c_{1}{ }^{2} c_{2}{ }^{2} k^{6} t^{3} e^{\frac{b k x}{a}}+864 a^{2} b^{2} k^{2} t c_{1} e^{\frac{b k x}{3 a}} \\
& +432 a^{2} b^{2} k^{2} t c_{2} e^{\frac{b k x}{6 a}}-24 a b^{4} k^{4} t^{2} c_{2} e^{\frac{b k x}{6 a}}+864 a^{2} b^{2} k^{2} t c_{1} e^{\frac{b k x}{a}}+1296 a^{2} b^{2} k^{2} t c_{1}^{2} e^{\frac{2 b k x}{3 a}} \\
& -432 a^{2} b^{2} k^{2} t c_{2}{ }^{3} e^{\frac{b k x}{2 a}}-216 a^{2} b^{2} k^{2} t c_{2}{ }^{4} e^{\frac{2 b k x}{3 a}}-48 a b^{4} k^{4} t^{2} c_{2}{ }^{2} e^{\frac{b k x}{3 a}}-24 a b^{4} k^{4} t^{2} c_{2}{ }^{3} e^{\frac{b k x}{2 a}} \\
& +216 a^{2} b^{2} k^{2} t c_{1}{ }^{4} e^{\frac{4 b k x}{3 a}}-2 b^{6} c_{1} c_{2}{ }^{2} k^{6} t^{3} e^{\frac{2 b k x}{3 a}}-96 a b^{4} c_{1} c_{2}{ }^{2} k^{4} t^{2} e^{\frac{2 b k x}{3 a}} \\
& +1296 a^{2} b^{2} c_{1}{ }^{2} c_{2} k^{2} t e^{\frac{5 b k x}{6 a}}-432 a^{2} b^{2} c_{1} c_{2}{ }^{3} k^{2} t e^{\frac{5 b k x}{6 a}}+432 a^{2} b^{2} c_{1}{ }^{3} c_{2} k^{2} t e^{\frac{7 b k x}{6 a}} \\
& -72 a b^{4} c_{1}{ }^{2} c_{2} k^{4} t^{2} e^{\frac{5 b k x}{6 a}}-24 a b^{4} c_{1} c_{2}{ }^{3} k^{4} t^{2} e^{\frac{5 b k x}{6 a}}-24 a b^{4} c_{1}{ }^{3} c_{2} k^{4} t^{2} e^{\frac{7 b k x}{6 a}} \\
& \left.\left.-48 a b^{4} c_{1}{ }^{2} c_{2}{ }^{2} k^{4} t^{2} e^{\frac{b k x}{a}}+1296 a^{2} b^{2} c_{1} c_{2} k^{2} t e^{\frac{b k x}{2 a}}-72 a b^{4} c_{1} c_{2} k^{4} t^{2} e^{\frac{b k x}{2 a}}\right)\right\} \\
& /\left(62208 a^{4}\left(c_{1} e^{\frac{b k x}{3 a}}+c_{2} e^{\frac{b k x}{6 a}}+1\right)\right)^{6}
\end{aligned}
$$

The results are given in the following figures and table: 


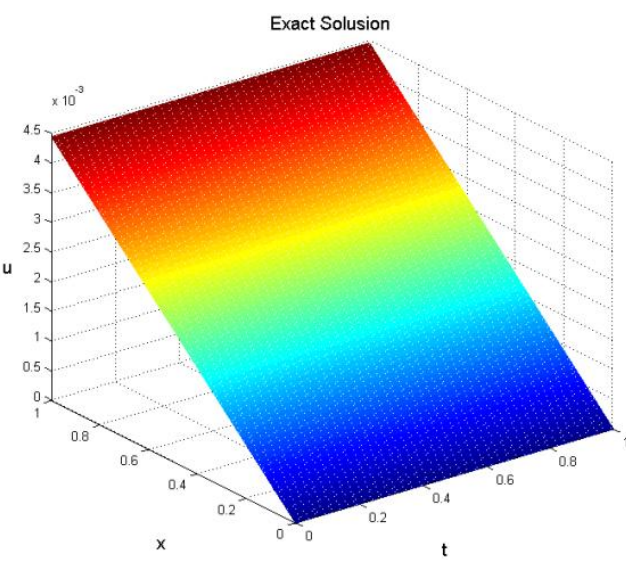

Show the (Exact) solution for $\mathrm{c}_{1}=\mathrm{c}_{2}=1, \mathrm{a}=\mathrm{b}=\mathrm{k}=0.2$

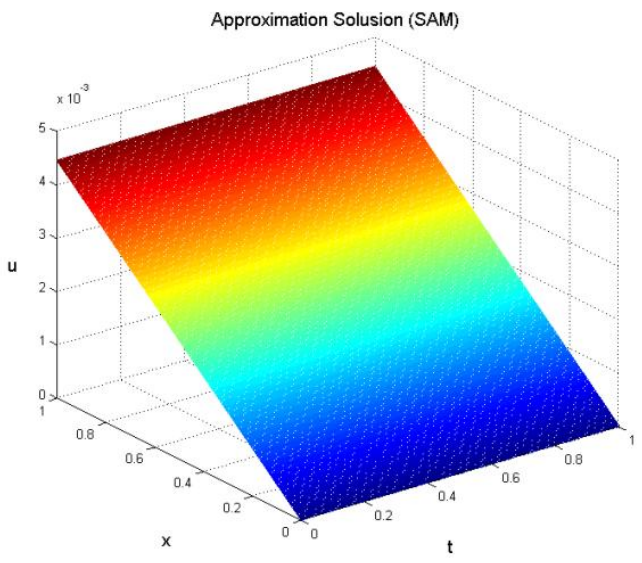

Show the (SAM) solution for $\mathrm{c}_{1}=\mathrm{c}_{2}=1, \mathrm{a}=\mathrm{b}=\mathrm{k}=0.2$

Table 1: The numerical results for the approximate solutions obtained by SAM in comparison with the exact solutions when $\mathrm{c}_{1}=\mathrm{c}_{2}=1, \mathrm{k}=0.2, \mathrm{a}=0.2, \mathrm{~b}=0.2$

\begin{tabular}{|c|c|c|c|c|}
\hline $\operatorname{Space}(x)$ & $\operatorname{Time}(t)$ & Exact & SAM & Error \\
\hline \multirow{4}{*}{0} & 0 & 0 & 0 & 0 \\
\hline & 0.4 & 0 & 0 & 0 \\
\hline & 0.8 & 0 & 0 & 0 \\
\hline & 1 & 0 & 0 & 0 \\
\hline \multirow{4}{*}{0.4} & 0 & 0.001777725104909530 & 0.001777725104909530 & $2.1684 \mathrm{E}-19$ \\
\hline & 0.4 & 0.001777567087241040 & 0.001777567087242080 & $1.0404 \mathrm{E}-15$ \\
\hline & 0.8 & 0.001777409055529230 & 0.001777409055537550 & 8.3223E-15 \\
\hline & 1 & 0.001777330034408260 & 0.001777330034424510 & $1.6254 \mathrm{E}-14$ \\
\hline \multirow{4}{*}{0.8} & 0 & 0.003555134221294890 & 0.003555134221295000 & $4.3368 \mathrm{E}-19$ \\
\hline & 0.4 & 0.003554818270210270 & 0.003554818270212350 & $2.0804 \mathrm{E}-15$ \\
\hline & 0.8 & 0.003554502291036530 & 0.003554502291053180 & $1.6641 \mathrm{E}-14$ \\
\hline & 1 & 0.003554344290918590 & 0.003554344290951090 & $3.2501 \mathrm{E}-14$ \\
\hline \multirow{4}{*}{1} & 0 & 0.004443621597267900 & 0.004443621597267900 & 0 \\
\hline & 0.4 & 0.004443226737372940 & 0.004443226737375540 & $2.5995 \mathrm{E}-15$ \\
\hline & 0.8 & 0.004442831842364240 & 0.004442831842385040 & 2.0798E-14 \\
\hline & 1 & 0.004442634381695170 & 0.004442634381735790 & $4.0620 \mathrm{E}-14$ \\
\hline
\end{tabular}

\section{CONCLUSION}

Successive Approximations Method used to solve nonlinear diffusion equation with convection term. Fig.(1) and Fig.(2) shows the comparison between the exact solution and the numerical solution obtained by Successive Approximations Method (SAM), For $c_{1}=1, c_{2}=1, \mathrm{k}=0.2$, $\mathrm{a}=0.2$, and $\mathrm{b}=0.2$.It can be seenthat the solution obtained by the present method is nearly identical with that given by exact solution. The absolute error $\left|u_{\text {exact }}(x, t)-u_{2}(x, t)\right|$ of example be observed in Table (1).and showed that the SAM is closed to the exact solution, also this method is suitable for this kind of problem.

\section{REFERENCES}

[1] F. Brauer and J. A Nohel, Ordinary Differential Equations, ( a First Course, $2^{\text {nd }}$ edition, W. A. Benjamin, INC. London, 1973).

[2] L.Debnath, Nonlinear Partial Differential Equations for Scientists and Engineers, (Birkhauser, Boston, ,1997).

[3] R. Cherniha and M. Servo, Lie and non-lie symmetries of nonlinear diffusion equations with convection term, Symmetry in Nonlinear Mathematical Physics, 2, 444-449,1997.

[4] A. J., Jerri, Introduction To Integral Equations With Applications, (Marcel Dekker, Inc, New York and Basel,1985).

[5] R. K., Saeed, Computational methods for solving system of linear Volterra and integro-differential equations, Ph.D. Thesis, Salahaddin University/Erbil, Iraq, 2006.

[6] E. A., Coddinggton, An introduction To Ordinary Differential Equations With Applications, (PrenticsHall,INC., 1961) .

[7] V.F Zaitsev and A.D. Polyanim, Handbook of Nonlinear Partial Differential Equations,(Chapman and Hall/CRC Press, Boca Raton, 2004). 